\title{
The Magnetosphere as a Complex System
}

\author{
J. A. Valdivia ${ }^{\mathrm{a}, \mathrm{b}}$ J. Rogan ${ }^{\mathrm{a}}$ V. Muñoz ${ }^{\mathrm{a}}$ L. Gomberoff ${ }^{\mathrm{a}}$ \\ A. Klimas ${ }^{c}$ D. Vassiliadis ${ }^{\mathrm{d}}$ S. Sharma ${ }^{\mathrm{e}}$ B. Toledo ${ }^{\mathrm{a}}$ \\ L. Wastavino ${ }^{\text {a }}$ \\ ${ }^{a}$ Departamento de Fisica, Facultad de Ciencias, Univerisidad de Chile, Las \\ Palmeras 3425, Nuñoa, Santiago, Chile \\ ${ }^{\mathrm{b}}$ Departamento de Fisica, Universidad de los Andes, Cra. 1E No. 18A-10. Edificio \\ H. A.A. 4976, Bogotá D.C, Colombia. \\ ${ }^{\mathrm{c}}$ NASA/Goddard Space Flight Center, Greenbelt, MD 207r1, USA \\ ${ }^{\mathrm{d}}$ Universities Space Research Association, at NASA/Goddard Space Flight Center, \\ Greenbelt, MD 207r1, USA. \\ e Department of Astronomy, University of Maryland, College Park, MD 20740, \\ USA
}

\begin{abstract}
The magnetosphere is a complex system, with multi-scale spatio-temporal behavior. Self-organization is a possible solution to two seemingly contradicting observations: (a) the repeatable and coherent substorm phenomena, and, (b) the underlying selfsimilar turbulent behavior in the plasma sheet. Such states, are seen to emerge naturally in a plasma physics model with sporadic dissipation, through spatio-temporal chaos.
\end{abstract}

Key words: Self-organizations, Space Plasmas, Substorms

\section{Introduction}

Recent observations seem to suggest that plasmas, under certain conditions, demonstrate very complex behavior, that includes self-similar behavior, emergence and self-organization, phase transitions, turbulence, spatio-temporal

1 This project has been financially supported by FONDECyT under contracts $\mathrm{N}^{\circ} 1030727, \mathrm{~N}^{\circ} 1050350$, and $\mathrm{N}^{\circ} 1030957$.

2 Corresponding author: J. A. Valdivia, alejo@fisica.ciencias.uchile.cl 
chaos, and so on (Lu and Hamilton, 1991; Carreras et al., 1996; Boffeta et al., 1999; Klimas et al., 2000; Tangri et al., 2003; Valdivia et al., 2003; Borovsky et al., 2003).

A number of driven models are providing new tools for the study of complex behavior in plasmas. For example, Bak et al. (1987) proposed a discrete stochastically driven model of a sandpile that displays self-similar event behavior when driven by an asymptotically small driving force. This means that the self-similar behavior is present only when the small grains are driven into the system with a time scale that goes to infinity, i.e., the system dissipates completely before a new small grain is dropped into the system. Even though the dynamics at small scales are complex, displaying the characteristic $1 / f$ type of behavior, the global state of the sandpile is coherent and repetitive. It is coherent in the sense that once it is established, at the largest scales the overall average shape of the sandpile does not change much, even though it displays complicated and unpredictable behavior at the smaller scales when driven. It is repetitive and robust in the sense that it is the state to which the system converges regardless of the initial condition and of any tuning of parameters, except that the driving force is asymptotically small (Jensen, 1998; Hwa et al., 1992). Similar models have been used to describe plasma dynamics (Lu and Hamilton, 1991; Carreras et al., 1996; Chapman et al., 1998; Takalo et al., 1999a,b; Vassiliadis et al., 1998). Starting from a more physical point of view, a number of "physics based" models have been constructed in an effort to disentangle the nature of plasma turbulence. Here is appropriate to mention the fluid and MHD shell models (Biskamp, 2000), that have served as a framework to study the nonlinear dynamics of systems with large Reynolds numbers. Even though these models are a clear simplification of the complete equations, they are claimed to represent the energy flow in a qualitative manner (Boffeta et al., 1999; Biskamp, 2000). Klimas et al. (2000) and Valdivia et al.(2003) derived from the plasma equations, but using anomalous localized dissipation, a continuous model of magnetic annihilation that displays selfsimilar event behavior, reminiscent of a self-organized critical state. A similar model was postulated earlier as a possible continuous representation of a sandpile (Lu, 1995). These models, which have advanced considerably from the first discrete stochastic models proposed by Bak et al. (1987), can display a global self-organized state with self-similar event behavior under completely deterministic, spatio-temporal chaotic, conditions.

Therefore, we can observe that there is an increasing need to clarify the conceptual framework in which to study the relationship between self-organization and spatio-temporal chaotic dynamics, and between stochasticity and deterministic dynamics. We don't necessarily see them as opposing paradigms, but in some situations may very well be related. The study of self-organization and spatio-temporal chaotic dynamics, and their relation with turbulence is a subject at the forefront of astrophysics and space research. 


\section{Complexity in the magnetosphere}

In the magnetosphere, there are two seemingly contradicting observations. First, the magnetotail plasma sheet appears to be a dynamic and turbulent region (Borovsky et al., 1997; Ohtani et al., 1998). Second, the complex behavior of the plasma sheet, in particular in the regions associated with substorm onset, is at first sight difficult to reconcile with the predictability of the geomagnetic indices (Vassiliadis et al., 1995; Valdivia et al., 1996) and the coherence and repeatability of the substorm cycle that has led to the identification of its distinct phases (Klimas et al., 1996; Baker et al., 1999). Therefore, it is natural to ask about the significance of the complexity observed in the magnetosphere, and the relationship with the two paradigms mentioned above. Such realizations, have led us immediately to new and exciting modeling techniques that may help us describe some of the complex behavior observed in the magnetosphere, and more generally, in the astrophysical setting.

Indeed, Borovsky et al. (1997) have found strong evidence that the plasma sheet is a high Reynolds number plasma, showing strong turbulence around 20 Earth radius (RE) tailward of Earth at all activity levels. This region is close to the expected position of the near-Earth neutral line (NENL), therefore, the reconnection and related instabilities thought to be crucial for the neutral line formation process, and even the production of the magnetospheric substorm, may be affected. Similar turbulent magnetic field fluctuations have also been measured far in the magnetotail ( 200 RE) (Hoshino et al., 1994). Closer to the Earth $(<10 \mathrm{RE})$ observations of magnetic fluctuations, associated with substorm onset, seem to be due to a system of turbulent and chaotic filamentary electric currents (Ohtani et al., 1998). The results of Borovsky et al. (1997) and Ohtani et al. (1998) are in sharp contrast to the standard picture of plasma sheet transport with laminar earthward flow in a well ordered magnetic field. Instead they are more consistent with the presence of elementary transport events, probably bursty bulk flows, that are accelerated in local reconnection regions that subsequently brake when they reach regions of higher pressure, if they travel Earthward. These BBFs (Baumjohan et al., 1990; Angelopoulos et al. , 1992; Angelopoulos et al., 1994, 1996) are characterized by high-speed flow bursts, plasma vortices, and strongly varying magnetic field (Fairfield, 1998).

Angelopoulos et al. (1999) went further and studied the nature of the intermittent properties of these BBFs, and their event statistics. On the basis of previous observations, Sergeev et al. (1996) have postulated impulsive dissipation events, localized in space and time, which are the manifestations of sporadic tail reconnection. In a study of many substorm onsets with Geotail observations, Nagai et al. (1998) found that at the position of the spacecraft magnetic reconnection lasts for approximately $10 \mathrm{~min}$, stopping even while the 


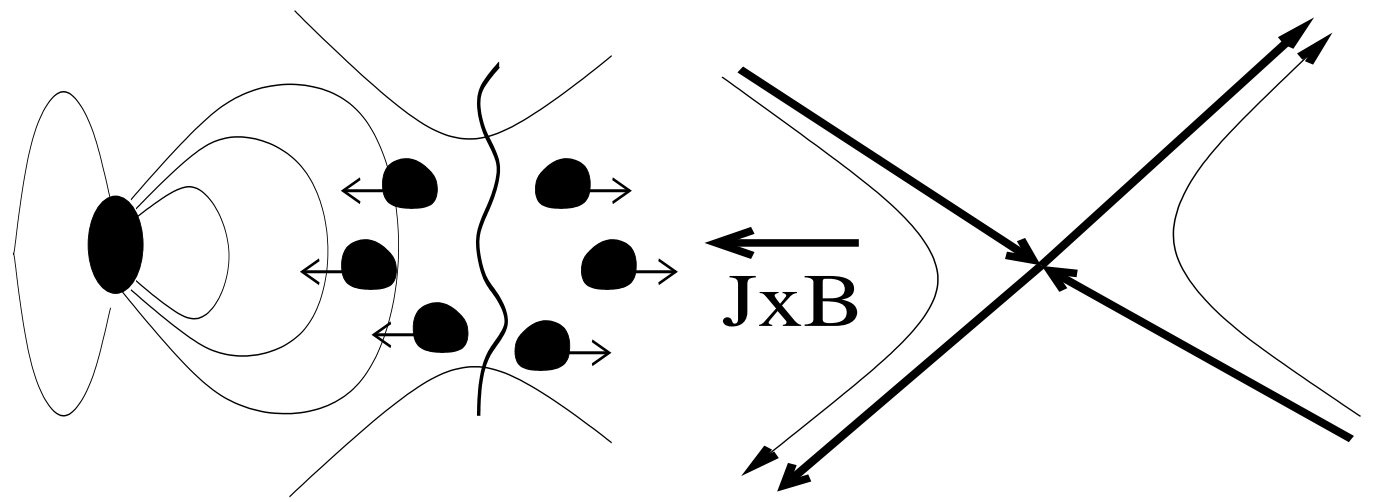

Fig. 1. (a) The conceptual view of the complex magnetosphere. Somewhere in the tail bursty flows are generated, which transport plasma and magnetic flux. This transport seems to permit global topological change during substorms. (b) These plasma burst can in principle be accelerated at localized reconnection sites with interesting magnetic topologies. In this picture we have a possible acceleration region with the magnetic field lines in an x configuration and the current perpendicular to the local plane of the picture.

substorm expansion phase proceeds. Thus, implying that reconnection occurs at localized sites that turn on and off over the course of a substorm. These observations suggest an intermittent magnetotail with localized dissipation, as pictured in Fig. 1a, where bursts of plasma are accelerated at some localized sites in the magnetotail. Figure 1b shows a possible localized acceleration region, with each blob in Fig. 1a generated at one of these localized acceleration region (Chang, 2002).

On the other hand, there is mounting indirect evidence that a non-equilibrium complex state with self-similar event statistics really exists in the magnetosphere. We have taken 2 years of AL index time series data, as shown in Fig. 2, which represents the lowest value of the horizontal component of the magnetic field measured at 10 or more stations distributed longitudinally in the northern hemisphere auroral zone. The AL index is in a certain way related to the dissipation of energy in the auroral region. We can see that the time series has periods of intermittent behavior, asides from a year modulation. The power spectrum for this data set is shown in Fig. $2 \mathrm{~b}$ and consists of two power-law spectral regions separated by a break as suggested by Tsurutani et al. (1990) and Consolini et al. (1997). This break seems to be related to a physical time scale of about $2 \mathrm{hrs}$ (Takalo et al, 1994), that is associated with the typical duration of an isolated substorm.

The spectral brake in the power spectrum of the AL index has also been related to the nonlinear behavior of substorms and have been studied in detail by many authors (Borovsky et al., 1993; Tsurutani et al., 1990; Vassiliadis et al., 1995; Valdivia et al., 1996). 

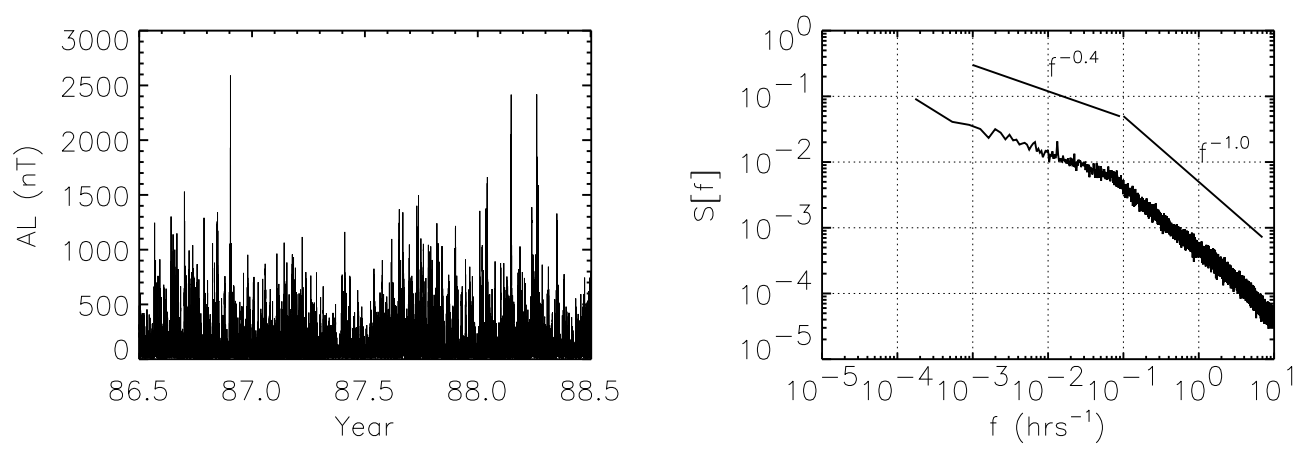

Fig. 2. (a) The -AL time series for a 2 year period. Note that there seems to be a yearly periodicity. (a) The power spectrum of the time series. The resolution of the time series is 1 minute.

Consolini (1997) took the AL index as a global representation of the energy dissipation, and showed a well-defined power law distribution for "burst strength" events for over 4 decades, (Uritsky andPudovkin, 1998). An important feature of many systems that exhibit self-organization is that they dissipate their stored energy in "avalanches" characterized by power-law distributions for avalanche size and duration, and by a power spectra associated with the internal energy dissipation. For the AL time series, these periods of dissipation can be characterized, in a first approach, by the "energy dissipated" $\Delta E$ (area under the curve), the time duration $\Delta t$, and the time separation between dissipations $\Delta \theta$ as described in Fig. 3a. We have constructed the event distribution for energy, duration and time between dissipations as shown in Figs. 3b-d respectively. The event distributions seem to display clear power law behavior, at least for a range of variables.

Lui et al. (2000) went even further with this idea and studied the spatial event statistics of ionospheric energy dissipation from Polar UVI images. They found power law distributions over many decades except for large events. But Uritsky et al. (2003) showed that this power law distribution extends even to large events when the spatio-temporal event statistics are considered, that is, the dissipation events need to be integrated not only in space, but also in time. Note that this spatio-temporal integration is exactly what is done to reconstruct self-similar event statistics in sandpiles. This is probably the best up-to-date indirect evidence that the magnetotail is in a self-organized global state. McPherron and Hsu (1998) compared the timing information from each Pi2 pulsation (impulsive oscillations of the geomagnetic field with amplitudes of about $1 \mathrm{nT}$ in the frequency range of $5-30 \mathrm{mHz}$ ) to other indicators of substorm onset and suggested that the pulsations correspond to local plasma sheet regions becoming temporarily unstable, but their effect is quenched before it can affect global stability. Hence the growth phase does not evolve monotonically towards onset, but goes through several metastable or even locally unstable states. This is strikingly similar to the conditions required for 


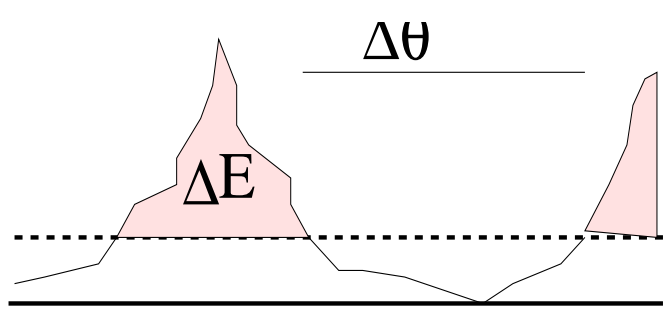

$\Delta \mathrm{t}$

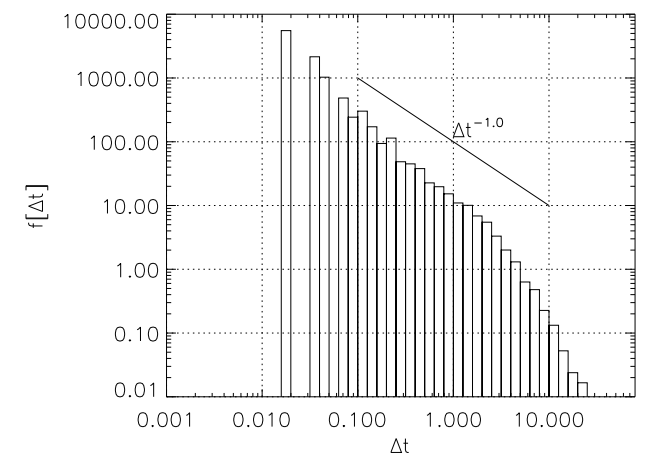

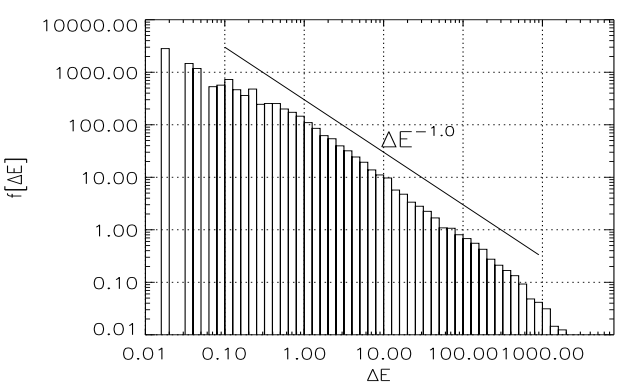

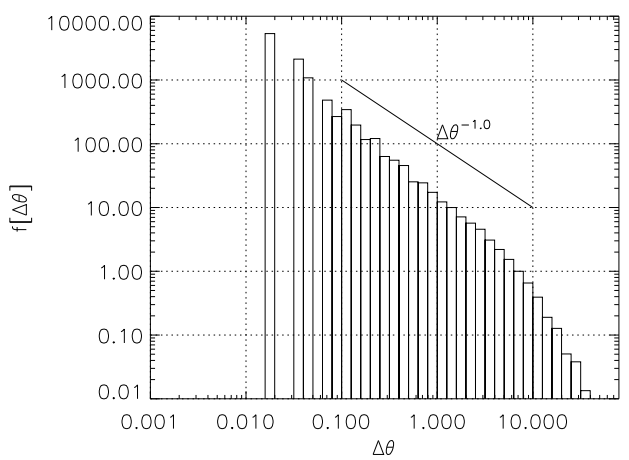

Fig. 3. (a) Schematic description of the intermittent dissipation used in the construction of the event distributions. (b) The distribution of the "energy" dissipated, $\Delta E$, during the event. (c) The distribution of the event duration, $\Delta t$. (d) The distribution of the time between events, $\Delta \theta$. Using a threshold value of $-A L>100 n T$ to define an event, we obtain $N=10365$ events. The distributions are constructed by counting the number of events $N_{i}$ in exponentially increasing bins, but normalized by the exponentially increasing size of the bin, i.e., a normalized $\widehat{N}_{i}$. A probability of events is constructed by dividing $\widehat{N}_{i}$ by $\sum_{i} \widehat{N}_{i}$. The effective number of events in a given bin is finally obtained by multiplying by the total number of events. Note that there are bins for which there are less that one effective event, and this measure can be taken as an indication that this particular bin is not statistically significant. The variation of some of the parameters does not change the results significantly. Time values are in minutes.

a system to develop self-organized criticality Lu (1995).

The existence of a strongly turbulent plasma sheet in the regions where substorm onset is thought to occur is, at first sight, difficult to reconcile with the global coherence and repeatability of the substorm cycle that has led to the identification of its distinct phases (Klimas et al., 1996; Baker et al., 1999), unless the self organized state is invoked. Furthermore, modern input-output methods have been quite successful in modeling and/or predicting the geomagnetic activity of the magnetosphere (Vassiliadis et al., 1995; Valdivia et al., 1996, 1999; Klimas et al., 1997). Similarly, low-dimensional physics based models (Klimas et al., 1992; Horton et al., 1998) have been shown to replicate many of the features of the solar wind-magnetosphere interaction over very long intervals of electrojet index data. It is difficult to understand how a dynamical system containing a strongly turbulent key component can ex- 
hibit indications of low dimensional behavior. But Chang (1992, 1998, 1999) has shown that this is exactly the behavior to be expected from a system in self-organization and studies of turbulent transport in magnetically confined plasmas have confirmed this (Diamond and Hahm, 1995; Newman et al., 1996; Carreras et al., 1996).

Thus, we propose to study the magnetospheric substorm phenomenon as an "avalanche" of many small reconnection events in the turbulent plasma sheet under the assumption that it is in, or near, a self-organized global state. At, or near, substorm onset the plasma sheet can be described by a self-organized global state containing significant small scale turbulence. Even though this self-organized global state is a dynamical state in nature, with superimposed unpredictable behavior, it is described as a unique global state that is inevitable, and repeatable. This statement is true of self-organized critical sandpile models (Bak et al., 1987) as well. This is the basis, we think, for the global coherence and repeatability of the substorm phenomenon in the turbulent plasma sheet. In the self-organized state, events occur over a broad distribution of spatial and temporal scales, ranging from localized events, which we associate with flow bursts, to "system wide" events, substorms, in which a significant portion of the plasma sheet is lost in the form of plasmoids.

Sitnov et al. (2000) has shown that the magnetospheric dynamics during substorms, under certain circumstances, shares a number of properties with nonequilibrium phase transitions. This is an intriguing result that we may investigate in detail within our present framework.

\section{Theoretical Background}

If the plasma sheet is in a state of self-organization, then understanding selforganization may be the key to understanding substorm onset. A quantitative characterization of that global self-organized state is a primary goal of our pro-

posed research. Within this characterization, special effort will be devoted to study the relationship between self-organization and spatio-temporal chaotic dynamics, and between stochasticity and deterministic dynamics.

It is extremely important here not to confuse self-organized state (SO) of a plasma with the more traditional self-organized critical (SOC) state of the sandpile models of Bak et al., (1987). Even though we may expect that the plasma dynamics, including turbulent plasma flows and magnetic field fluctuations, are different than the traditional sandpile dynamics, at the end they may belong to the same universality class. The SO state is characterized by critical behavior with power-law power spectra and scale invariant events with self-similar spatial structure and fractal topology. In this respect, Milovanov et 
al. (1996) has suggested a plasma sheet with a fractal current structure, while Antonova et al. (1998) proposed that the turbulent transport is necessary for the stability of the plasma sheet.

Lu (1995) postulated a set of criteria that would be required for a system to reach a $\mathrm{SO}$ state, which seem to be satisfied in the plasma sheet. There are a number of examples of self-organization in the literature (Jensen, (1998); and references therein), where the self-organized critical model of sandpiles is probably one of the most famous (Bak et al., 1987, 1988). These models have been very useful in strengthening our intuition about SO states. It is worth mentioning that the self organized state is a robust final state (it does not depend on the details of the dynamics and the physics of the system) and occurs in a variety of plasma (Lu and Hamilton, 1991; Vlahos et al., 1995; Diamond and Hahm, 1995; Carreras et al., 1996) and fluid environments. Also, it appears that the statistical behavior of many complex distributed systems is more a property of their self-organized state, if it is achieved, than the details of the physical processes that allow such state. It is probable that the statistics of substorms, pseudobreakups, and even the evolutions of the growth and expansion phases, are unrelated to the details of the reconnection process (Shay et al., 1998) other than that reconnection allows for the establishment of a self organized state.

Some of the statistical properties of the magnetospheric observations can be reproduced by discrete sandpile-like models (Chapman et al., 1998; Takalo et al., 1999a,b, 2001), which have pointed the way to more sofisticated modeling efforts.

We are interested in continuing some of the recent efforts to develop plasma physics models that evolve naturally into a self-organized state (Klimas et al., 2000; Valdivia et al., 2003), with self-similar events statistics. In particular, we propose to study the effect of intermittent reconnection and turbulence in the generation of a global self-organized state in plasma situations. Issues of relevance include, among others, the statistics of bursty flow acceleration, the distinction between self-generated and externally driven turbulence, and the characterization of stochasticity and deterministic dynamics.

\section{Modeling}

We now turn to a possible modeling approach to describe the ideas proposed up to here. In particular, we are interested in going beyond the statistical description of discrete sandpile-like models (Chapman et al., 1998; Takalo et al., 1999a,b, 2001) and formulate a plasma physics-based description that can account for the magnetospheric observations. We start from the following 
neutral plasma equation

$$
\begin{aligned}
\left(\partial \mu / \partial t+U_{j} \partial \mu / \partial x_{j}\right) & =-\mu \nabla \times \mathbf{U} \\
\mu\left(\partial \mathbf{U} / \partial t+U_{j} \partial \mathbf{U} / \partial x_{j}\right) & =\mathbf{J} \times \mathbf{B}-\nabla P+\nu \nabla^{2} \mathbf{U} \\
\left(\partial P / \partial t+U_{j} \partial P / \partial x_{j}\right) & =-\gamma P \nabla \cdot \mathbf{U}+(\gamma-1) \mathbf{J} \cdot(\mathbf{E}+\mathbf{U} \times \mathbf{B})-\nabla \mathbf{Q} \\
\partial \mathbf{B} / \partial t & =-\nabla \times \mathbf{E}
\end{aligned}
$$

and Ohm's law

$$
\mathbf{E}+\mathbf{U} \times \mathbf{B}=\eta \mathbf{J}+\alpha_{1} \mathbf{J} \times \mathbf{B}+\alpha_{2} \frac{\partial \mathbf{J}}{\partial t}+\alpha_{3} \nabla \mathbf{P}_{\mathbf{e}}+\ldots
$$

We assume $\alpha_{1}=\alpha_{2}=\alpha_{3}=0, \nabla \mathbf{Q}=0$, and $B=\nabla \times A$.

A first simplification to the problem can be achieved by constructing a 1-D field annihilation model from Faradays' law, with $\mathbf{A}=A_{y}(z, t) \widehat{y}$

$$
\frac{\partial A_{y}(z, t)}{\partial t}=\eta \frac{\partial^{2} A_{y}(z, t)}{\partial z^{2}}+S(z, t)
$$

where the current density $J=-\partial^{2} A_{y} / \partial z^{2}$ and $S$ corresponds to $(\mathbf{U} \times \mathbf{B})_{y}$. This deceivingly simple diffusion equation will provide the starting point to understand the complex behavior required to establish a global SO state. Klimas et al., (2000) introduced (see Lu 1995) sporadic dissipation through a spatio-temporal evolution for $\eta$ as

$$
\frac{d \eta}{d t}=\frac{(q(J)-\eta)}{\tau} \quad q(J)= \begin{cases}\eta_{\max } & |J|>J_{c} \\ \eta_{\min } & |J|<\beta J_{c}\end{cases}
$$

The sporadic dissipation is achieved through the trigger function $\mathrm{q}$, at a given position, which is defined by a hysteretic loop. In this sense q will remain in the low state $q=\eta_{\text {min }}$, until $|J|>J_{c}$ where it undergoes a transition to the high state $q=\eta_{\max }$. Similarly, when in the high state, $\mathrm{q}$ will not make the transition to the low state $q=\eta_{\min }$ until $J<\beta J_{c}(\beta<1)$. We take $-L \leq z \leq L$, with $L=20, \Delta x=0.1, S(z)=S_{o} \operatorname{Cos}(\pi z / 2 L), J_{c}=0.04$, $\eta_{\max }=5$ (normalized to $\left.c^{2} L V_{a} / 4 \pi\right), V_{a}$ a reference Alfven's speed, $\tau=1$, $\eta_{\min }=10^{-10}, S_{o}=3 \times 10^{-5}$, and $J=0$ at the boundaries. We expect that this 1-D simple model would apply in the region given by the dark line of Fig. 4 , which would represent the magnetic diffusion region of the magnetotail.

We start with $A_{y}(z)=0$ and we let the system evolve. If we take $\eta=$ const, then $J(x, t)=-\left(S_{o} / \eta\right) \operatorname{Cos}(\pi z / 2 L)$ is the solution to Eq. 3. A steady solution 


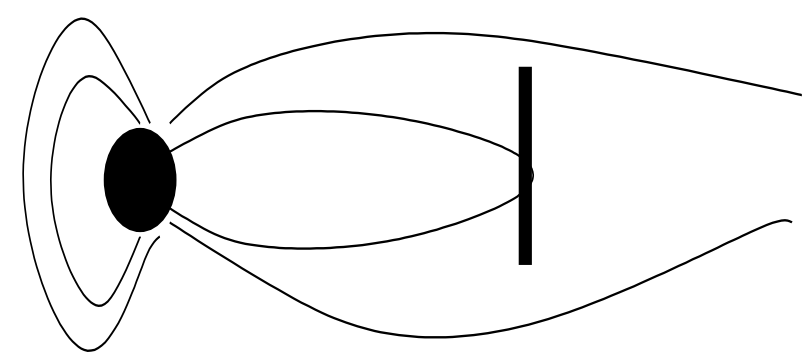

Fig. 4. Location we expect the 1-D field annihilation model to apply.

is permitted as long as $S_{o}<J_{c} \eta_{\min }$ or in theory for $S_{o}>J_{c} \eta_{\max }$. If we define the energy as $E(t)=\int B(x, t)^{2} d x / 2$, then

$$
\frac{d E}{d t}=-F+I
$$

with the essentially constant input rate $I(t)=\int S(x) B(x, t) d x$. The sporadic dissipation rate $F(t)=\int \eta(x, t) J(x, t)^{2} d x$ for the case $S_{o}<J_{c} \eta_{\min }$ is $F=$ $S_{0}^{2} L^{2} \eta_{\min }$

In the following we are interested in showing the situation for $S_{0}>J_{c} \eta_{\min }$ and we intend to study the event statistics of the collective effects of many such interacting instability sites, derived from observations and data analysis, in a complementary manner to microphysics and particle kinetics. Hence we use this nonlinear resistivity in an attempt to characterize some of the complex microphysics behavior, with q acting like a physical current driven instability (Papadopoulos et al., 1985) with a threshold $J_{c}$ that is higher than the value required to maintain the instability.

Let's consider the case of $\beta=0.9$. As an example of the rich behavior displayed by this model, we show $B_{x}$ and $\eta$ in Fig. 5a and Fig. 5b respectively in the intermediate range $J_{c} \eta_{\min }<S_{0}<J_{c} \eta_{\max }$. Even though the system can display complex behavior with strong underlying spatio-temporal variability, its global state, as described by $B_{x}$, is robust, coherent and repetitive. This may provide a hint about the coherent and repetitive behavior of the magnetosphere during substorms. The sporadic dissipation rate $F(t)=\int \eta(x, t) J(x, t)^{2} d x$ is shown in Fig. 5c, due to the spatio-temporal dissipation $\eta J^{2}$ given in Fig. 5 d.

Due to the boundary conditions, and the reflection symmetry of the example given above, we can compute the spatial mode decomposition of $J(z, t)$ from

$$
J_{m}(t)=\frac{1}{L} \int_{-L}^{L} J_{y}(z, t) \cos \left(\frac{\pi m z}{2 L}\right) d z
$$


as shown in Fig. 5e and Fig. $5 \mathrm{f}$ for an instance where $F$ is low (namely $t=20$ in Fig. 5c) and high respectively (namely $t=50$ in Fig. 5c). It is interesting to observe that the higher modes become active during the dissipation events, Fig. 5f, as compared with the quiet times, Fig. 5e.
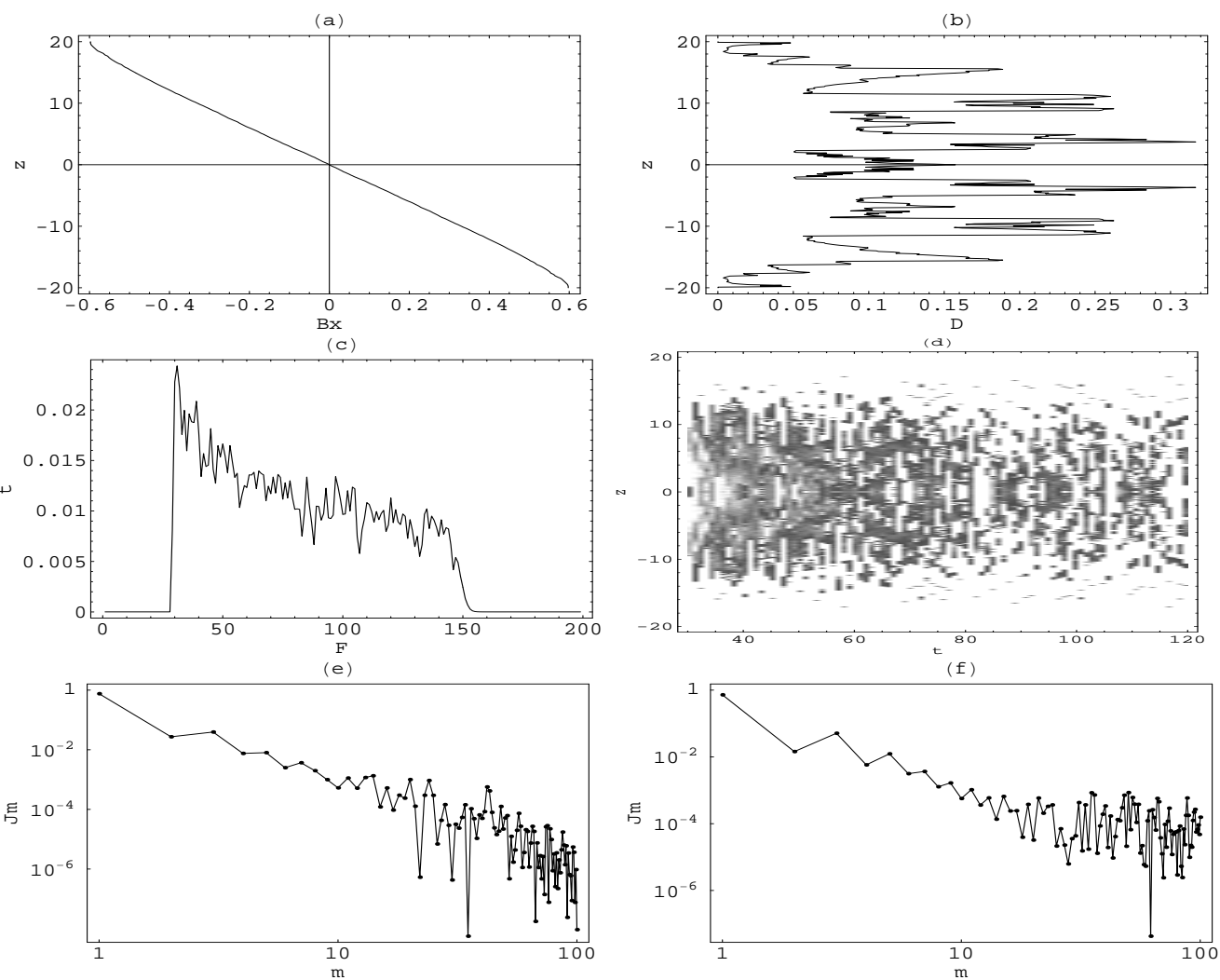

Fig. 5. Spatial profile of (a) $B_{x}$ and (b) $\eta$ for $\beta=0.9$ (at time defined by arrow in (c)). (c) $\mathrm{F}(\mathrm{t})$. (d) The spatio-temporal evolution of $\eta J^{2}$ in gray scale. (e) The mode expansion of the current $J_{m}$ at $\mathrm{t}=20$. (f) The mode expansion of the current $J_{m}$ at $\mathrm{t}=50$.

Using a long time series of $\mathrm{F}$ (Fig. 6a) we compute the event statistics of energy dissipated (Fig. 6b) and duration (Fig. 6c). The statistics seem to be insensitive to the threshold value used to define an event, namely $F=F_{\min }=$ $10^{-5}$. $S_{0}$ was chosen in the intermediate region $J_{c} \eta_{\min }<S_{0}<J_{c} \eta_{\max }$. There is a range of values of $\Delta E$ and $\Delta t$ for which the distribution of events seems to display self-similar behavior.

A power law distribution for event duration of BBFs in the plasma sheet has been found by Angelopoulos et al. (1999), and Fig. 6c is suggestive of if. Of course in this model we have not introduced the plasma evolution yet.

This complexity emerges from purely deterministic spatio-temporal chaotic dynamics, with well defined loading-unloading cycles. In practice the robust critical behavior occurs only in a range of $S_{0}$ with $S_{p}<S_{0}<S_{c}$. The phase 

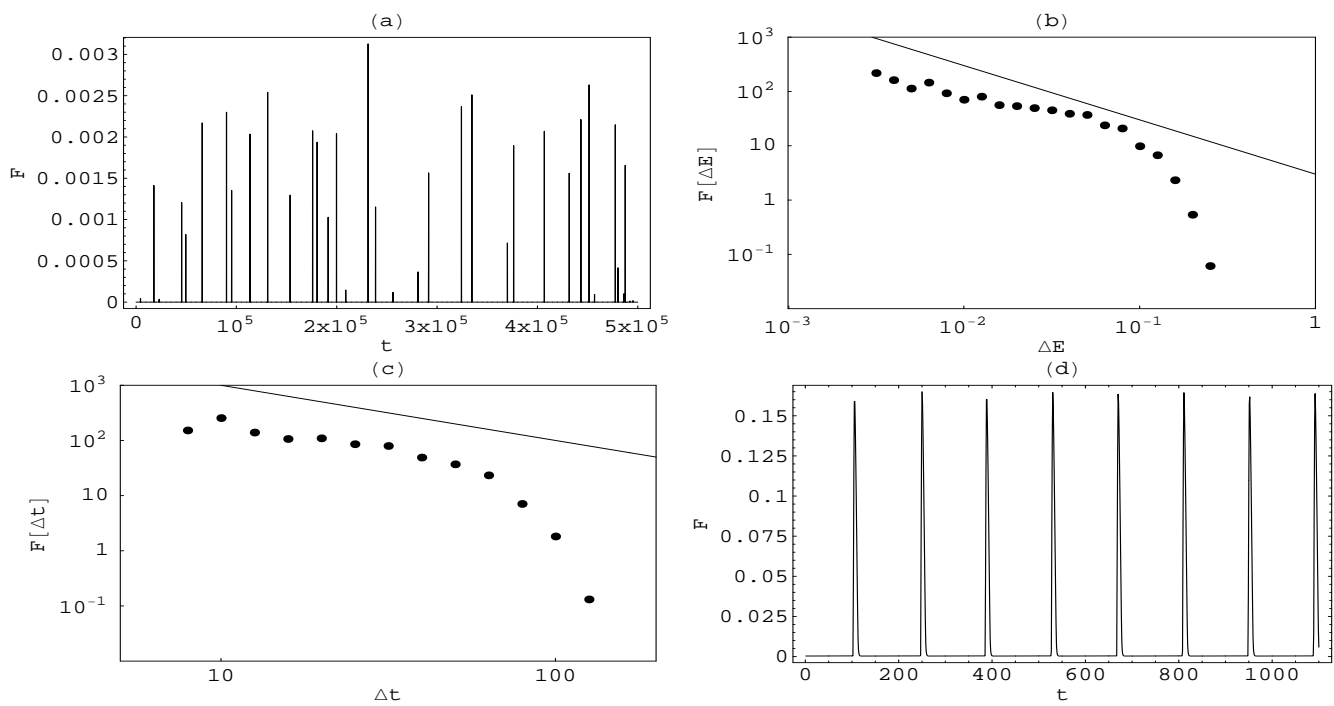

Fig. 6. (a) $\mathrm{F}(\mathrm{t})$ for a long integration time using $\beta=0.9$. The event distribution of (b) energy dissipation and (c) duration. The event statistics are constructed using a logarithmic histogram with a log bin size of 0.1. This is then normalized by the linear size of each bin. (d) $\mathrm{F}(\mathrm{t})$ for a long integration time using $\beta=0.5$ displaying quasiperiodic behavior. It is not exactly periodic when the spatial structure is observed, there are small differences from dissipation to dissipation.

diagram for the parameter $S_{0}$ is shown in Fig. 7 , with the following definitions

(1) for $S_{0}<J_{c} \eta_{\min }$ we have a steady state.

(2) for $J_{c} \eta_{\min }<S_{0}<S_{p}$ we have in principle a quasiperiodic situation, as can be described by $F(t)$ (see Fig. $6 \mathrm{~d}$ for $\beta=0.5$ ). The existence of this regime depends strongly of the ratio $\eta_{\min } / \eta_{\max }$ (Tangri et al., 2003). These oscillations may be related to some periods in which the magnetosphere seems to be oscillating, i.e., saw-tooth like oscillations (see Fig. $6 \mathrm{~d}$ for $\beta=0.5$ ).

(3) for $S_{p}<S_{0}<S_{c}$ we can have the SO state with a robust critical behavior that displays self-similar statistics. As $S_{0} \rightarrow S_{c}$ the duration of the loading cycle is about the same as the duration of the unloading cycle. Uritsky et al. $(2001 \mathrm{a}, 2002)$ found that $S_{c}=2 \times 10^{-2} J_{c} \eta_{\text {max }}$ for $\beta=0.9$ and $\eta_{\text {min }}=0$. Therefore, this model clearly departs from standard sandpile models in which critical behavior is only realized in the limit $S_{o} \rightarrow 0$ (Vespignani and Zapperi, 1998).

(4) for $S_{c}<S_{0}<J_{c} \eta_{\max }$ we have a chaotic behavior but without the loadingunloading cycle. In essence the time separation between cycles tend to zero as $S_{0} \rightarrow S_{c}$.

(5) for $S_{0}>J_{c} \eta_{\max }$ we have a situation in which $J \sim$ const almost everywhere (except boundaries). It is important not to over extrapolate from this model, but the transition at $S_{o} \sim J_{c} \eta_{\max }$ is similar to a first order phase transition, and may provide an explanation for the observation of Sitnov et al. (2000) (Uritsky et al., 2001b). This would correspond to the 
situation where the magnetosphere is directly driven, with the dissipation in direct response with the driver. The internal dynamics are not as relevant as in the previous regimes.

The actual existence of these regimes depend on the values of $\beta$ and $\eta_{\text {min }}$ as controlling factors. This qualitative description of the bifurcation diagram is shown in Fig. 7. It turns out that the hysteresis is present in virtually all SO models, including sandpiles, and it is the reason behind the existence of the loading-unloading cycle necessary for the intermittent behavior. This hysteresis is also present in deterministic and stochastic systems that evolve into intermittent behavior without threshold conditions. These issues are very important and must be studied in details.

We are currently studying the bifurcation diagram of this 1-D spatio-temporal chaotic system using a more dynamical approach (e.g., Boffeta et al. (2002)). In particular we are interested in the the transition from steady state to fully developed spatio-temporal chaos. The result will be presented elsewhere.

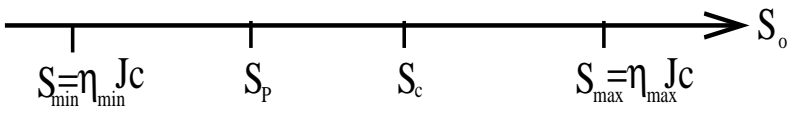

Fig. 7. The phase diagram representing the possible dynamical behaviors as a function of the driver amplitude $S_{0}$. The actual existence of these regimes depend on the values of $\beta$ and $\eta_{\min }$ as controlling factors.

The 1-D model is in general a dynamical model for the magnetic field in the diffusion region of the magnetotail. It is expected that in such a diffusion region, the magnetic field annihilated energy is transfered to the plasma in an intermittent manner. Therefore, we expect that burst of plasmas should be generated through a $J \times B$ force close to the localized dissipation regions, see Fig. 1a. In fact Chang (1999) postulated that the multiscale turbulence in the magnetotail evolves into a SO state where the locally, $J \times B$, accelerated plasma interacts with the turbulent field and plasma around it generating bursts reminiscent of BBFs. The SO state evolves precisely when the turbulent field and bursty plasma generate coherent structures. We will include the plasma evolution in the model, but for that we need to construct a 2-D model as described by Eqs. 1, including the dynamical $\eta$. It incorporates selfconsistently the turbulent flow velocity and the magnetic field. The question is whether it evolves into a SO state. Since we do not examine the kinetic details, we model the plasma sheet as a magnetofluid. This does not mean that MHD can account for the microphysics of the plasma sheet. We solve Eq. 1 in two dimensions, but with the dissipation given by Eq. 4. The geometry of the system is similar to Ugai and Tsuda (1977) (Petschek, 1964; Petschek and Thorne, 1967; Sonnerup, 1970) in which we have a symmetric system at both $x=0$ and $z=0$, with an imposed constant inflow z-velocity $U_{z, o}$ and magnetic field $B_{o}$ at $z=L_{z}$. We have outgoing conditions at $x=L_{x}$. Figure 8 a shows the region where this $2-\mathrm{D}$ model is expected to apply. 
Let's first assume that we don't have hysteresis, hence $\beta \rightarrow 0$, with a constant $\eta=\eta_{\max }$. This a situation similar to the one discussed in Biskamp (2000). There is the formation of a thin current sheet as the incoming plasma gets transported out of the system, as shown in Fig. 8b. If we now include the hysteretic dissipation term, $\beta=0.9$, we can study how the plasma turbulence enhances or quenches the transport. Figures $8 \mathrm{c}, \mathrm{d}$ show the turbulent current density, at different times, with underlying spatio-temporal diffusion. We can already see that even though we have strong underlying turbulence, there is a well defined global state that permits the dissipation and transport of energy through the system, but in a bursty fashion. The details of the characterization of this system will be published elsewhere.

(a)

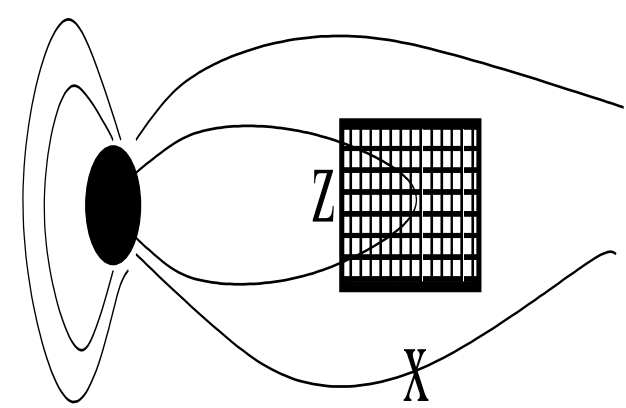

(c)

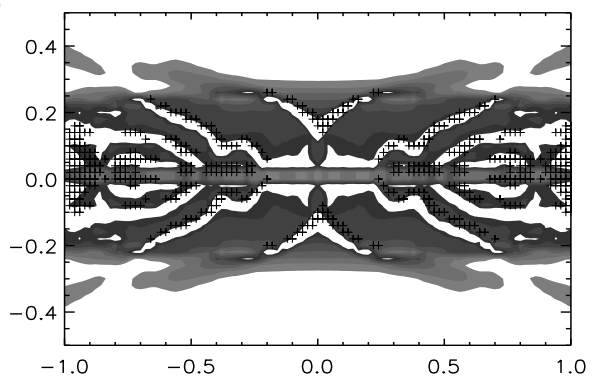

(b)

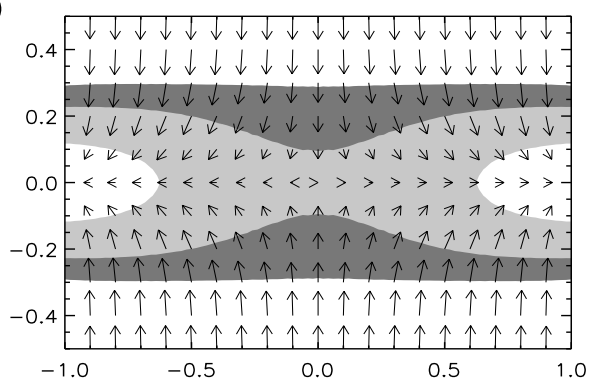

(d)

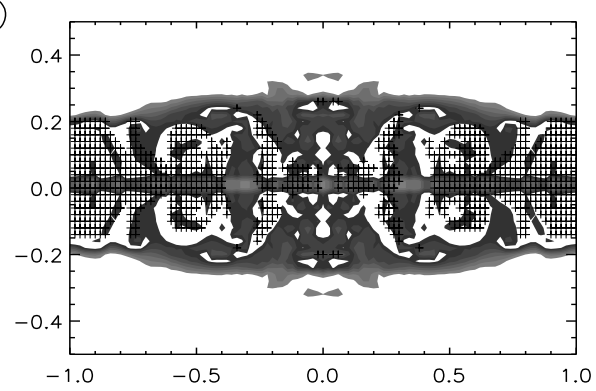

Fig. 8. (a) The region where the 2-D model is expected to apply. (b)The evolution of the current sheet model when $\eta=$ const. The current density $\mathrm{J}$ is also shown at two different times in (c), (d) respectively for the dynamical $\eta$ situation, with $\beta=0.9$.

\section{Conclusions}

Physical plasmas, under certain conditions, seems to demonstrate very complex behavior, from self-organization to spatio-temporal chaos to phase transitions, etc. While deterministic chaotic models are providing a new tool for studying turbulent behavior in plasmas, the emergence of a self-organized state allows for the possibility of a repeatable coherent global state, with underlying complex dynamics at all scales. In this needed framework, it is necessary to 
clarify the emergence of turbulent behavior from stochastic and deterministic spatio-temporal dynamics.

In the magnetosphere the emergence of a self-organized state from the underlying turbulence observed in the plasma sheet becomes a plausible solution to the seemingly contradicting observation of the repeatability and coherent substorm phenomena with underlying turbulent and complex behavior in the plasma sheet. In this work, we assumed a simple parameterization of the dissipation $\eta$ to study the collective effect of many interacting localized instability sites in a complementary manner to microphysics and particle kinetics. Even though the exact details of the microphysics (ballooning, cross field current, variant of tearing, etc. (Pellat et al., 1991; Lee and Wolf, 1992; Lui et al., 1993; Hurricane et al., 1995; Lui et al., 1995; Buchner et al., 1999)) cannot be accounted by such simple parameterizations, it appears that the statistical behavior of many complex distributed systems is more a property of their self-organized state, if it is achieved, than the details of the physical processes that allow such state. This is a general characteristic of systems that are close to criticality where many systems belong to the same universality class, suggesting that it is probable that the statistics of substorms, pseudobreakups, and even the evolutions of the growth and expansion phases, are unrelated to the details of the dissipation process (Shay et al., 1998) other than that dissipation allows for the establishment of a self-organized state.

We proposed a simple 1-D model, from a physics based framework, that seems to evolve into a self-organized state with underlying complex behavior. This 1-D model already has some of the properties observed in the magnetospheric dynamics, and it is expected that the proposed sporadically dissipative 2-D plasma model may help us bring some light about the relationship between the energy dissipation with the self-similar complex behavior observed in the magnetosphere.

Acknowledgements: This project has been financially supported by FONDECyT under contracts $\mathrm{N}^{\circ} 1030727, \mathrm{~N}^{\circ} 1050350$, and $\mathrm{N}^{\circ} 1030957$.

\section{References}

Angelopoulos, V., W. Baumjohann, C. F. Kennel, F. V. Coroniti, M. G. Kivelson, R. Pellat, R. J. Walker, H. Lühr, G. Paschmann, Bursty Bulk Flows in the Inner Central Plasma Sheet, J. Geophys. Res., 97(A4), 4027-4039, 10.1029/91JA02701, 1992.

Angelopoulos, V., C. F. Kennel, F. V. Coroniti, R. Pellat, M. G. Kivelson, R. J. Walker, C. T. Russell, W. Baumjohann, W. C. Feldman, J. T. Gosling, Statistical characteristics of bursty bulk flow events, J. Geophys. Res., 99(A11), 21257-21280, 10.1029/94JA01263, 1994. 
Angelopoulos, V., F. V. Coroniti, C. F. Kennel, M. G. Kivelson, R. J. Walker, C. T. Russell, R. L. McPherron, E. Sanchez, C. -. Meng, W. Baumjohann, G. D. Reeves, R. D. Belian, N. Sato, E. Friis-Christensen, P. R. Sutcliffe, K. Yumoto, T. Harris, Multipoint analysis of a bursty bulk flow event on April 11, 1985, J. Geophys. Res., 101(A3), 4967-4990, 10.1029/95JA02722, 1996.

Angelopoulos V., Mukai T., Kokubun S., Evidence for intermittency in Earth's plasma sheet and implications for self-organized criticality, Phys. Plasmas, 6, 4161-4168, 1999.

Antonova, E. E., M. V. Stepanova, M. V. Teltzov, B. A. Tverskoy, Multiple inverted-V structures and hot plasma pressure gradient mechanism of plasma stratification, J. Geophys. Res., 103(A5), 9317-9332, 10.1029/97JA03090, 1998.

Bak, P., C. Tang, K. Wiesenfeld, Self-organized criticality: an explanation of 1/f noise, Phys. Rev. Lett., 59, 381-384, 1987.

Bak, P., C. Tang, K. Wiesenfeld, Self-organized criticality, Phys. Rev. A., 38, 364-374, 1988.

Baker, D. N., T. I. Pulkkinen, J. Büchner, A. J. Klimas, Substorms: A global instability of the magnetosphere-ionosphere system, J. Geophys. Res., 104(A7), 14601-14612, 10.1029/1999JA900162, 1999.

Baumjohann, W., G. Paschmann, H. Lühr, Characteristics of high-speed ion flows in the plasma sheet, J. Geophys. Res., 95(A4), 3801-3809, 10.1029/89JA02923, 1990.

Birn, J., Computer Studies of the Dynamic Evolution of the Geomagnetic Tail, J. Geophys. Res., 85, 1214-1222, 1980.

Biskamp, D., Magnetic Reconnection in Plasmas, Cambidge University Press, Cambridge, 2000.

Boffeta G., V. Carbone, P. Giuliani, P. Veltri, and A. Vulpiani, Power Laws in Solar Flares: Self-Organized Criticality or Turbulence?, Phys. Rev. Lett., 83, 4662-4665, 1999.

Boffeta G., M. Cencini, M. Falcioni, A. Vulpiani, Predictability: a way to characterize complexity, Physics Reports, 356, 367-474, 2002.

Borovsky, J. E., R. J. Nemzek, R. D. Belian, The occurrence rate of magnetospheric-substorm onsets: Random and periodic substorms, J. Geophys. Res., 98(A3), 3807-3814, 10.1029/92JA02556, 1993.

Borovsky, J. E., R. C. Elphic, H. O. Funsten, and M. F. Thomsen, The Earth's plasma sheet as a laboratory for flow turbulence in high-beta MHD, J. Plasma Phys., 57, 1-34, 1997.

Borovsky, and H. O. Funsten, Role of solar wind turbulence in the coupling of the solar wind to the Earth's magnetosphere, J. Geophys. Res, 108, 12461270, doi:10.1029/2002JA009601, 2003.

Buchner, J. E., Kinetic effects controlling the onset of 3-D tail reconnection, in Substorms-4: International Conference on Substorms-4, Lake Hamana, Japan, March 9 13, 1998, Astrophys. Space Sci. Libr., vol. 238, edited by S. Kokubun and Y. Kamide, p. 461-466, Terra Sci., Tokyo, 1998. 
Carreras, B, D. Newman, V. E. Lynch, P. H. Diamond, A model realization of self-organized criticality for plasma confinement, Phys. Plasmas, 3, 29032911, 1996.

Chang, T., Low-dimensional behavior and symmetry breaking of stochastic system near criticality: Can these effects be observed in space and in the laboratory? IEEE Trans. Plasma. Phys., 20, 691-694, 1992.

Chang, T., Sporadic localized reconnections and multiscale intermittent turbulence in the magnetotail, in Geospace Mass and Energy Flow, No. 104 in Geophysical Monograph, edited by J. L. Horwitz, D. L. Gallagher, and W. K. Peterson (American Geophysical Union, Washington, D.C.), 193-200, 1998.

Chang, T., Self-organized criticality, multi-fractal spectra, sporadic localized reconnections and intermittent turbulence in the magnetotail, Phys. Plasmas, 6, 4137-4145, 1999.

Chang, T., Private communication, 2002.

Chapman, S., et al., A simple avalanche model as an analogue for geomagnetic activity, Geophys. Res. Lett., 25, 2397-2400, 1998.

Consolini, G, Sandpile cellular automata and magnetospheric dynamics, in Cosmic Physics in the year 2000, ed. By S. Aiello, N. Lucci, G. Sironi, A. Treves, and U. Villante, Proc. Of VIII GIFCO Conference, 123-127, Italian Physical Society, Bologna, 1997.

Fairfield, D. H., T. Mukai, A. T. Y. Lui, C. A. Cattell, G. D. Reeves, T. Nagai, G. Rostoker, H. J. Singer, M. L. Kaiser, S. Kokubun, A. J. Lazarus, R. P. Lepping, M. Nakamura, J. T. Steinberg, K. Tsuruda, D. J. Williams, T. Yamamoto, Geotail observations of substorm onset in the inner magnetotail, J. Geophys. Res., 103(A1), 103-118, 10.1029/97JA02043, 1998.

Diamond, P. H., and T. S. Hahm, On the dynamics of turbulent transport near marginal stability, Phys. Plasmas, 2, 3640-3649, 1995.

Hayashi, T, T. Sato, Magnetic reconnection: Acceleration, heating, and shock formation, J. Geophys. Res., 83, 217-220, 1978.

Horton, W., I. Doxas, A low-dimensional dynamical model for the solar wind driven geotail-ionosphere system, J. Geophys. Res., 103(A3), 4561-4572, 10.1029/97JA02417, 1998.

Hoshino, M., A. Nishida, and T. Yamamoto, Turbulent magnetic field in the distant magnetotail: Bottom-up process of plasmoid formation?, Geophys. Res. Lett., 21, 2935-2938, 1994.

Hurricane, O. A., R. Pellat, F. V. Coroniti, A new approach to low-frequency

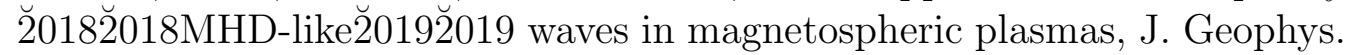
Res., 100(A10), 19421-19428, 10.1029/95JA01523, 1995.

Hwa, T., and M. Kardar, Avalanches, hydrodynamics and discharge events in models of sandpiles, Phys. Rev. E, 45, 7002-7023, 1992.

Jensen, H. J., Self-organized criticality, Cambridge Lecture notes in Physics, Cambridge University Press, Cambridge, UK., 1998.

Klimas, A. J., D. N. Baker, D. A. Roberts, D. H. Fairfield, J. Buchner, A nonlinear dynamical analogue model of geomagnetic activity, J. Geophys. 
Res., 97(A8), 12253-12266, 10.1029/92JA00794, 1992.

Klimas, A. J., D. Vassiliadis, D. N. Baker, D. A. Roberts, The organized nonlinear dynamics of the magnetosphere, J. Geophys. Res., 101(A6), 1308913114, 10.1029/96JA00563, 1996.

Klimas, A. J., D. Vassiliadis, D. N. Baker, Data-derived analogues of the magnetospheric dynamics, J. Geophys. Res., 102(A12), 26993-27010, 10.1029/97JA02414, 1997.

Klimas, A. J., J. A. Valdivia, D. Vassiliadis, D. N. Baker, M. Hesse, J. Takalo, Self-organized criticality in the substorm phenomenon and its relation to localized reconnection in the magnetospheric plasma sheet, J. Geophys. Res., 105(A8), 18765-18780, 10.1029/1999JA000319, 2000.

Lee, D.-Y., R. A. Wolf, Is the Earth's magnetotail balloon unstable?, J. Geophys. Res., 97(A12), 19251-19257, 10.1029/92JA00875, 1992.

Lu, E. T., and R. J. Hamilton, Avalanches and the distribution of solar flares, Astrophys. J., 380, L89-L92, 1991.

Lu, E. T., Avalanches in continuum driven dissipative systems, Phys. Rev. Lett, 74, 2511-2514, 1995.

Lui, A. T. Y., P. H. Yoon, Quasi-linear analysis of ion Weibel instability in the Earth's neutral sheet, J. Geophys. Res., 98(A1), 153-164, 10.1029/92JA02034, 1993.

Lui, A. T. Y., P. H. Yoon, Preliminary nonlocal analysis of cross-field current instability for substorm expansion onset, J. Geophys. Res., 100(A10), 1914719154, 10.1029/95JA01404, 1995.

Lui, A. T. Y., Chapman, S. C., Liou, K., Newell, P. T., Meng, C. I., Brittnacher, M., Parks, G. K., Is the dynamic magnetosphere an avalanching system? Geophys. Res. Lett. 27, 911-914, 2000 .

McPherron, R. L., T. Hsu, The main onset of the magnetospheric substorm, in Proc. of International conference on substorm-4, ed. by Y. Kamide, and S. Kokubun, Tokyo, Terra Scientific, 79-82, 1998.

Milovanov, A. V., L. M. Zelenyi, G. Zimbardo, Fractal structures and power law spectra in the distant Earth's magnetotail, J. Geophys. Res., 101(A9), 19903-19910, 10.1029/96JA01562, 1996.

Nagai, T., M. Fujimoto, Y. Saito, S. Machida, T. Terasawa, R. Nakamura, T. Yamamoto, T. Mukai, A. Nishida, S. Kokubun, Structure and dynamics of magnetic reconnection for substorm onsets with Geotail observations, J. Geophys. Res., 103(A3), 4419-4440, 10.1029/97JA02190, 1998.

Newman, D. E., et al., The dynamics of marginality and self-organized criticality as a paradigm for turbulent transport, Phys. Plasmas, 3, 1858-1866, 1996.

Ohtani, S., K. Takahashi, T. Higuchi, A. T. Y. Lui, H. E. Spence, J. F. Fennell, AMPTE/CCE - SCATHA simultaneous observations of substormassociated magnetic fluctuations, J. Geophys. Res., 103(A3), 4671-4682, 10.1029/97JA03239, 1998.

Petschek, H. E., Magnetic field annihilation, in the Physics of Solar Flares, the AAS-NASA Symp. on the Physics of Solar Flares, ed. W. N. Hess (Wash- 
ington DC:NASA), 425-439, 1964.

Petschek, H. E., Thorne R. M., The Existence of Intermediate Waves in Neutral Sheets, Astrophys. J., 147, 1157-1163, 1967

Papadopoulos, K., Microinstabilities and anomalous transport, in Collisionless Shocks in the Heliosphere: a tutorial review, ed. By R. G. Stone, and B. T. Tsurutani, vol 34, 59-90, Washington DC, American Geophysical Union, 1985.

Pellat, R., F. V. Coroniti, and P. L. Pritchett, Does ion tearing exist? Geophys. Res. Lett., 18, 143-146, 1991.

Sergeev, V. A., T. I. Pulkkinen, R. J. Pellinen, Coupled-mode scenario for the magnetospheric dynamics, J. Geophys. Res., 101(A6), 13047-13066, 10.1029/95JA03192, 1996.

Shay, M. A., J. F. Drake, R. E. Denton, D. Biskamp, Structure of the dissipation region during collisionless magnetic reconnection, J. Geophys. Res., 103(A5), 9165-9176, 10.1029/97JA03528, 1998.

Sitnov, M. I., A. S. Sharma, K. Papadopoulos, D. Vassiliadis, J. A. Valdivia, A. J. Klimas, D. N. Baker, Phase transition-like behavior of the magnetosphere during substorms, J. Geophys. Res., 105(A6), 12955-12974, 10.1029/1999JA00279, 2000.

Sonnerup, B. U.,Magnetic field re-connexion in a highly conducting incompressible fluid, J. Plasm Phys, 4, 161-174, 1970.

Takalo, J., J. Timonen, H. Koskinen, Properties of AE data and bicolored noise, J. Geophys. Res., 99(A7), 13239-13250, 10.1029/94JA00516, 1994.

Takalo, J., J. Timonen, A. Klimas, J. Valdivia, D. Vassiliadis, Nonlinear energy dissipation in a cellular automaton magnetotail field model, Geophys. Res. Lett, 26, 1813-1816, 1999.

Takalo, J., J. Timonen, A. Klimas, J. Valdivia, D. Vassiliadis, A coupled map model for the magnetotail current sheet, Geophys. Res. Lett, 26, 2913-2916, 1999.

Takalo, J., J. Timonen, A. Klimas, J. A. Valdivia, D. Vassiliadis, A coupled map as a model of the dynamics of the magnetotail current sheet, J. of Atmos. and Solar-Terrestrial Phys, 63, 1407-1414, 2001

Tangri, V., A. Das, P. Kaw, R. Singh, Continuum self-organized-criticality model of turbulent heat transport in tokamaks, Phys. Rev. Lett., 91, 0250011-025001-4, 2003

Tsurutani, B. T., et al., The nonlinear response of AE to the IMF Bz: A spectral break at 5 hours, Geophys. Res. Lett., 17, 279-282, 1990.

Ugai, M., T. Tsuda, Magnetic field-line reconnexion by localized enhancement of resistivity. Part 1. Evolution in a compressible MHD fluid, J. Plasma Phys., 17, 337-256, 1977.

Uritsky V.M., Pudovkin M.I. Low frequency 1/f-like fluctuations of the AEindex as a possible manifestation of self-organized criticality in the magnetosphere. Ann. Geophysicae, 16, 1580-1588, 1998.

Uritsky, V. M, A. J. Klimas, J. A. Valdivia, D. Vassiliadis, D. N. Baker, Stable critical behavior and fast field annihilation in a magnetic field reversal 
model, J. of Atmos. and Solar-Terrestrial Phys, 63, 1425-1433, 2001a

Uritsky, V. M., A. J. Klimas, D. Vassiliadis, Comparative study of dynamical critical scaling in the auroral electrojet index versus solar wind fluctuations, Geophys. Res. Lett., 28, 3809-3812, 2001b

Uritsky, V. M., A. J. Klimas, D. Vassiliadis, Multiscale dynamics and robust scaling in a continuum current sheet model, Phys. Rev. E, 65, 046113-1046113-5, 2002

Uritsky, V. M., A. J. Klimas, D. Vassiliadis, Evaluation of spreading critical exponents from the spatiotemporal evolution of emission regions in the nighttime aurora, Geophys. Res. Lett., 30 (15), 1813-1816, doi:10.1029/2002GL016556, 2003.

Valdivia, J. A., A. Sharma, K. Papadopoulos, Prediction of magnetic storms by nonlinear dynamical methods, Geophys. Res. Lett., 23, 2899-2902, 1996.

Valdivia, J. A., D. Vassiliadis, A. Klimas, Modeling the spatial structure of the high latitude magnetic perturbation and the related current system, Phys. of Plasmas, 6, 4185-4194, 1999.

Valdivia, J. A., A. Klimas, D. Vassiliadis, V. Uritsky, J. Takalo, Selforganization in a current sheet model, Space. Sci Rev., 107, 273-282, 2003.

Vassiliadis, D., A. J. Klimas, D. N. Baker, D. A. Roberts, A description of the solar wind-magnetosphere coupling based on nonlinear filters, J. Geophys. Res., 100(A3), 3495-3512, 10.1029/94JA02725, 1995.

Vassiliadis, D., A. Anastasiadis, M. Georgoulis, L. Vlahos, Derivation of solar flare cellular automata models from a subset of the Magnetohydrodynamic equations, Astroph. J. Lett., 509, L53-L56, 1998.

Vespignani, A., and S. Zapperi, How self-organized criticality works: A unified mean-field picture, Phys. Rev. E, 57(6), 6345-6362, 1998.

Vlahos, L., M Georgoulis, and P. Paschos, The statistical flare, Astron. and Astroph., 299, 897-911, 1995. 